\title{
Production of Thrombin Complexes with DNA Aptamers Containing G-Quadruplex and Different Duplexes
}

\author{
Spiridonova VA ${ }^{1 *}$, Glinkina $\mathrm{KA}^{2}$, Gainutdinov $\mathrm{AA}^{2}$ and Arutyunyan $\mathrm{AM}^{1}$ \\ ${ }^{1}$ Lomonosov Moscow State University, Belozersky Institute of Physico-Chemical Biology, Moscow, Russia \\ ${ }^{2}$ Lomonosov Moscow State University, Chemistry Department, Moscow, Russia
}

\begin{abstract}
Despite major advances in the understanding of the molecular mechanisms of thrombosis and the development of effective thrombolytic agents, arterial thrombosis remains a formidable clinical problem. A new class of direct thrombin inhibitors (aptamers), has been described recently. Aptamers, single-stranded oligonucleotides with a length of 30-60 nucleotides, exhibit high affinity and specificity towards any defined recognition target (protein). Aptamers are antibody analogs in terms of both specificity and affinity, with an apparent advantage of the former to being reproduced by anautomated chemical synthesis. Aptamers are routinely selected by SELEX technology (Systematic Evolution of Ligands by Exponential enrichment), which is thoroughly discussed elsewhere. The DNA aptamers to thrombin have a very highly ordered tertiary structure (G-quadruplex). A great number of the DNA aptamers to thrombin with sophisticated structure have been designed and widely investigated as potential thrombin inhibitors using traditionalmedicine tests (TT, PT, APPT). In this manuscript we suggest the different structures of aptamers, based on RE31 structure, so-called G-quadruplex, which are bound to short and long duplex. The RE31 aptamer had being previously shown to have an order of magnitude prolonged thrombintime in comparison to 15TBA aptamer. The purpose of the present study was to investigate the trunked aptamers to thrombin structures by CD spectroscopy and estimate the stability of the thrombin complexes with aptamers using electrophoresis in polyacrilamide gels. Our findings clearly show that both G-quadruplex and duplex domains of RE31 as well as trunked aptamers are strong effectors of aptamer complex stability. Using a set of oligonucleotide models derived from RE31 sequence, we have shown that the attached duplex domain of trunked aptamers retains the antiparallel unimolecular G-quadruplex topology seen for 15TBA.
\end{abstract}

Keywords: Thrombin; Aptamer; Blood coagulation; Thrombin inhibitor; Structure

\section{Introduction}

Despite major advances in understanding of the molecular mechanisms of thrombosis and developing effective thrombolytic agents, arterial thrombosis is still a serious challenge to theoretical and clinical medicine. In recent decades, new class of the direct thrombin inhibitors, based on nucleic acid aptamers, have been created [1]. Thrombin is a multifunctional serine proteinase of trypsin family that plays the key role in the hemostasis cascade. This enzyme initiates clotting by hydrolyzing fibrinogen and activating blood platelets. The dominant structural features of the thrombin include a) deep active site cleft, and b) two positively charged surfaces, referred to as exosites I and II. Exosite I is the fibrinogen-binding site, while exosite II is named the heparin binding site of thrombin.

Thrombin overproduction, which may cause dangerous diseases, such as apoplexy and heart attack, is a growing interest in effective medicines against intravascular thrombus formation.

Aptamers, single-stranded oligonucleotides about 30-60 bases long, feature high affinity and specificity toward their target molecules (proteins). In this respect, aptamers are analogs of antibodies but their obvious advantage is that they can be synthesized by an automated method and selected from the resulting sequence pool using the SELEX technology (Systematic Evolution of Ligands by Exponential enrichment) [2-10].

The first DNA aptamer to thrombin was selected by Bock et al. [1]. These authors initially studied only the 15-mer sequence found in most clones, named 15TBA (thrombin binding aptamer). 15TBA sequence consists of two planar G-tetrads linked by T-G-T loop and two short $\mathrm{T}$-T loops that take part in binding to exosite 1 of thrombin (according to X-ray data) [11]. A crucial role in determining structure, stability and biological properties of $\mathrm{G}$ - quadruplexes is played by $\mathrm{K}^{+}$ion $[12,13]$.
15TBA efficiently inhibits thrombin coagulation activity (in particular, doubles the thrombin time in human plasma) [1]. This finding stimulated interest in DNA aptamers, and subsequent research in many laboratories has resulted in selection of more effective aptamer-based thrombin inhibitors [4,14]. For instance, aptamer 31 TGT increases the clotting time from $19 \mathrm{sec}$ (normal) to $108 \mathrm{sec}$ [15]. These aptamers differ in molecular structure, but all of them contain the G-quadruplex sequence characteristic of 15TBA. According to NMR data and CD-spectroscopy, all antithrombin aptamers other than 15TBA (consisting of the G-quadruplex alone) contain additional complementary (duplex) nucleotide sequences attached to the ends of the quadruplex $[5,16]$.

As a rule, the aptamers with more sophisticated structure have a highly ordered tertiary structure consisting of G-quadruplex domain with additional double-stranded (duplex) sequences attached to its ends. One of such aptamers, named RE31, has been proved to prolong thrombin time by an order of magnitude, compared to the results obtained with the 15TBA aptamer (consisting of G-quadruplex alone) $[17,18]$.

Special studies using UV spectroscopy and thermodynamic

*Corresponding author: Vera A. Spiridonova, Senior Research Scientist, A.N Belozersky Institute of Physico-Chemical Biology, Moscow State University, 119992, Moscow, Russia, Tel: (495) 939-3149; Fax: (495) 939-3181; E-mail: spiridon@belozersky.msu.ru

Received November 25, 2013; Accepted January 03, 2014; Published January 07,2014

Citation: Spiridonova VA, Glinkina KA, Gainutdinov AA, Arutyunyan AM (2014) Production of Thrombin Complexes with DNA Aptamers Containing G-Quadruplex and Different Duplexes. J Nephrol Ther 4: 149. doi:10.4172/2161-0959.1000149

Copyright: ( 2014 Spiridonova VA, et al. This is an open-access article distributed under the terms of the Creative Commons Attribution License, which permits unrestricted use, distribution, and reproduction in any medium, provided the original author and source are credited. 
analysis have been performed to evaluate the effect of these duplexes on the stability of G-quadruplex [16].

To date, a large number of DNA aptamers to thrombin have been produced and evaluated for inhibitor activity using traditional tests for clotting times (TT, PT, APPT) $[4,14,17,18]$.

Most studies on aptamer-based thrombin inhibition have been performed with the 15TBA aptamer, evaluating its effect in tests for clotting time. However, the process of aptamer-thrombin complex formation and stability of this complex have not been studied sufficiently.

We have created a new DNA aptamer, named RE31, consisting of the G-quadruplex (15 nucleotides) and a duplex domain (6 pairs of complementary nucleotides) linked together by 4 nucleotides [18]. This aptamer inhibits thrombin activity much more efficiently than 15TBA, increasing the clotting time to $250 \mathrm{sec}$ (compared to $27 \mathrm{sec}$ in case of the latter). These results clearly support the idea that the duplex domain takes part in forming the complex with thrombin. Several variants of RE31 with a truncated duplex domain have been synthesized and their structure has been evaluated by CD spectra analysis, but the role of this domain in RE31 binding to thrombin has not yet been clarified.

In our study, truncated variants of RE31 have been produced to assess their antithrombin activity. Their structure has been analyzed by CD spectroscopy and the stability of their complexes with thrombin has been evaluated using polyacrylamide gel electrophoresis. The results show that the truncated variants of RE31 are strong effector molecules that inhibit thrombin activity, but their complexes with thrombin differ in stability. Analyzing a set of oligonucleotide models, derived from the RE31 sequence, it has been found that the duplex domains attached to the G-quadruplex domain in truncated variants did not alter its initial topology characteristic of the 15TBA aptamer.

The purpose of this study was to analyze the structure of the truncated variants of the RE31 aptamer and their complexes with thrombin.

\section{Materials and Methods}

Experiments were performed using inorganic salts of reagent and chemically pure grades (Russia), agarose type IV (Sigma, United States), acrylamide, N,N'-methylenebisacrylamide (Serva, Germany), N,N,N',N'-tetramethylethylenediamine (TEMED) (BioRad, United States), ammonium persulfate (Reanal, Hungary), xylene cyanol, bromopheol blue (BDH, United States), SYBR Green (Helicon, Russia),glycerol (Serva, Germany), $\left[\gamma^{-32} \mathrm{P}\right]$ ATP (OOO Lima, Institute of Bioorganic Chemistry, Moscow), T4 polynucleotide kinase (Fermentas, Lithuania), human $\alpha$-thrombin (Haematologic Technologies Inc., United States), and the following oligonucleotides synthesized by the phosphoramidite method (Sintol, Russia):

15-TBA:dGGTTGGTGTGGTTGG;

RE19:dTAGGTTGGTGTGGTTGGGG;

RE21:dGTAGGTTGGTGTGGTTGGGGC;

RE23:dCGTAGGTTGGTGTGGTTGGGGCG;

RE25:dACGTAGGTTGGTGTGGTTGGGGCGT;

RE27:dGACGTAGGTTGGTGTGGTTGGGGCGTC;

RE29:dTGACGTAGGTTGGTGTGGTTGGGGCGTCA;

RE31:dGTGACGTAGGTTGGTGTGGTTGGGGCGTCAC.

\section{Radioactive DNA labeling}

The oligonucleotides were labeled with ${ }^{32} \mathrm{P}$ by the standard procedure, using $\left[\gamma^{-}{ }^{32} \mathrm{P}\right]$-ATP [19]. The concentration of the isolated labeled aptamer was determined by measuring the optical density of the solution at $260 \mathrm{~nm}$.

\section{Preparation of aptamer-thrombin complexes}

Oligonucleotide samples were heated at $100^{\circ} \mathrm{C}$ for $2 \mathrm{~min}$, rapidly chilled on ice, and incubated with thrombin in $20 \mathrm{mM}$ HEPES-KOH buffer, $\mathrm{pH} 7.2$, containing $140 \mathrm{mM} \mathrm{NaCl}$ and $5 \mathrm{mM} \mathrm{KCl}$ (similar to the salt content of human blood). Incubation was performed at $4^{\circ} \mathrm{C}$ for no less than $60 \mathrm{~min}$. The oligonucleotide concentration in each experiment was the same, while the thrombin concentration was varied in a wide range. The aptamer-protein complexes were separated from unbound components by nondenaturing electrophoresis in 8\% PAAG in Trisborate buffer $\mathrm{pH}=8,2$ at $10 \mathrm{~mA}$ and $6^{\circ} \mathrm{C}$. Gels were stained with SYBR GREEN 1 fluorescent dye, and fluorescent and radioactively labeled complexes were detected in an FLA-3000 fluorescent image analyzer (FujiFilm, Japan). Images and numerical data were processed using programs GIMP, ImageJ, and OriginPro 8.1.

\section{Circular dichroism (CD) spectra analysis}

CD spectroscopy of aptamers was performed with a modified JobinYvon Mark V dichrograph (France) connected to an IBM computer with the Graphwork program developed at the Belozersky Institute of Physico-Chemical Biology, Moscow State University. The CD spectra in a wavelength range of $\lambda=240-340 \mathrm{~nm}$, at $\Delta \lambda=1 \mathrm{~nm} / \mathrm{sec}$, were recorded in a $1-\mathrm{cm}$ cell at $25^{\circ} \mathrm{C}$. The spectrum of the buffer without oligonucleotides was used as baseline. The result spectra were average from three measurements. Averaging was carried out using Graphwork program. The values of $\mathrm{CD}$ intensit $\Delta \varepsilon=\frac{\Delta \grave{A}}{\mathrm{cl}}$ ecalculated into molar $\mathrm{CD}$ coefficients $(\Delta \varepsilon)$ by the equation, where $\Delta \mathrm{A}$ is instrument reading; $\mathrm{C}$ is aptamer concentration, mole $/ \mathrm{L}$; and $\mathrm{L}$ is optical path length, $\mathrm{cm}$.

\section{Results and Discussion}

Characteristics of the new antithrombin DNA aptamer RE31 were compared to those of 31-TBA, 15-TBA, and some other aptamers
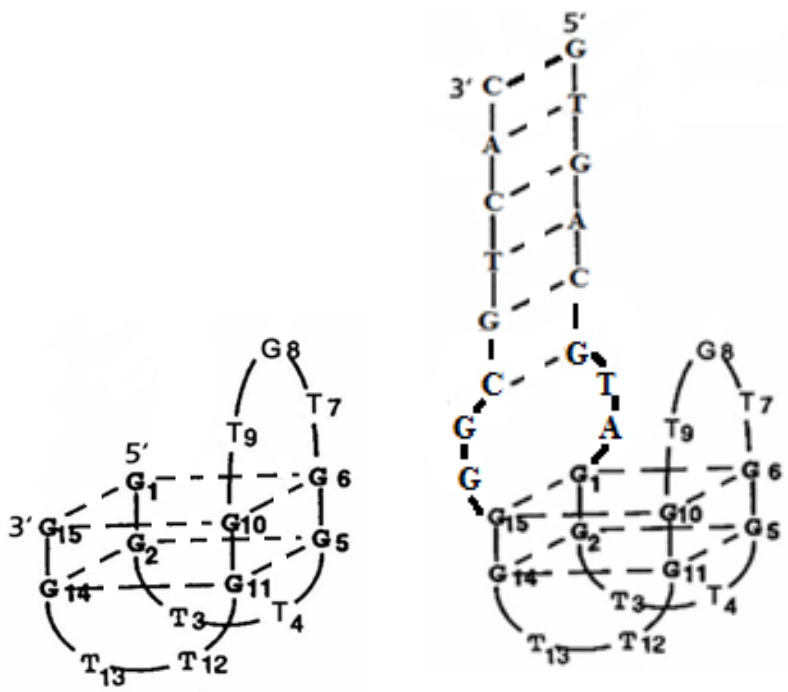

The structures include G-quadruplex pattern. The aptamer RE31 contains an additional duplex region, which is bounded to G-quadruplex with four nucleotides.

Figure 1: Proposed tertiary structures of the aptamers 15TBA (left) and RE31 (right). 
(Figure 1). Any of them inhibited thrombin clotting activity in human plasma, as followed from prolongation of the Thrombin Time (TT), Prothrombin Time (PT), and activated Partial Thromboplastin Time (aPTT). Measurements of TT are made to determine the rate of fibrin clot formation catalyzed by exogenous thrombin, while PT and aPTT values characterize the rate of clot formation catalyzed by endogenous thrombin formed as a result of stimulation of the blood clotting system. The plasma coagulation cascades evaluated in the PT ad aPTT tests are triggered via the exogenous and endogenous pathways respectively. The inhibitory activity of RE31 proved to be higher than that of 31TBA: similar effects (prolongation of plasma clotting time) in all three tests were observed at lower concentrations of RE31 (Figure 2). In our previous experiments, 31-TBA showed a stronger inhibitory activity than the short 15-TBA aptamer consisting of G-quadruplex alone [17]. Hence, we decided to analyze the activity and stability of truncated RE31 variants (Figure 3).

By analogy with similar oligonucleotides [16], it could be assumed that the structure of these aptamers includes both G-quadruplex and complementary sequences that can form a duplex domain. Indeed, the CD spectra of all molecules included in analysis proved to have a distinct peak at $294 \mathrm{~nm}$, which is characteristic of antiparallel G-tetrad structures; i.e., all of them contained the G-quadruplex domain (Figure
4). To estimate its stability in different aptamers, their CD spectra were recorded at temperatures increasing from 5 to $70^{\circ} \mathrm{C}$, at intervals of $5-7^{\circ} \mathrm{C}$. The results provided evidence for gradual degradation of the G-quadruplex upon such heating. The CD values of positive maximums recorded at $294 \mathrm{~nm}$ were used as a basis for plotting melting curves of all DNA aptamers and calculating melting temperatures of their G-quadruplex domains (Table 1).

As follows from Table 1, the attachment of additional oligonucleotide sequences to the G-quadruplex destabilizes its structure. On the other hand, the greater the number of complementary pairs formed by these sequences, the higher the stability of the G-quadruplex domain in a given aptamer. This domain is the least stable in aptamer RE25, where five pairs of nucleotides are attached to it. Three of them can form a duplex, but its stability is low.

The question arose as to whether the stability of G-quadruplex structure could have an effect on the formation of aptamer-thrombin complexes. To answer it, the reaction mixtures were resolved by electrophoresis in 8\% PAAG under nondenaturing conditions. Each experiment was performed in triplicate, and the formation of complexes with thrombin was recorded for every aptamer in the test series (Figure 5). After computer processing of electrophoretic data and gel images, isotherms of aptamer-thrombin binding were plotted and linearized in
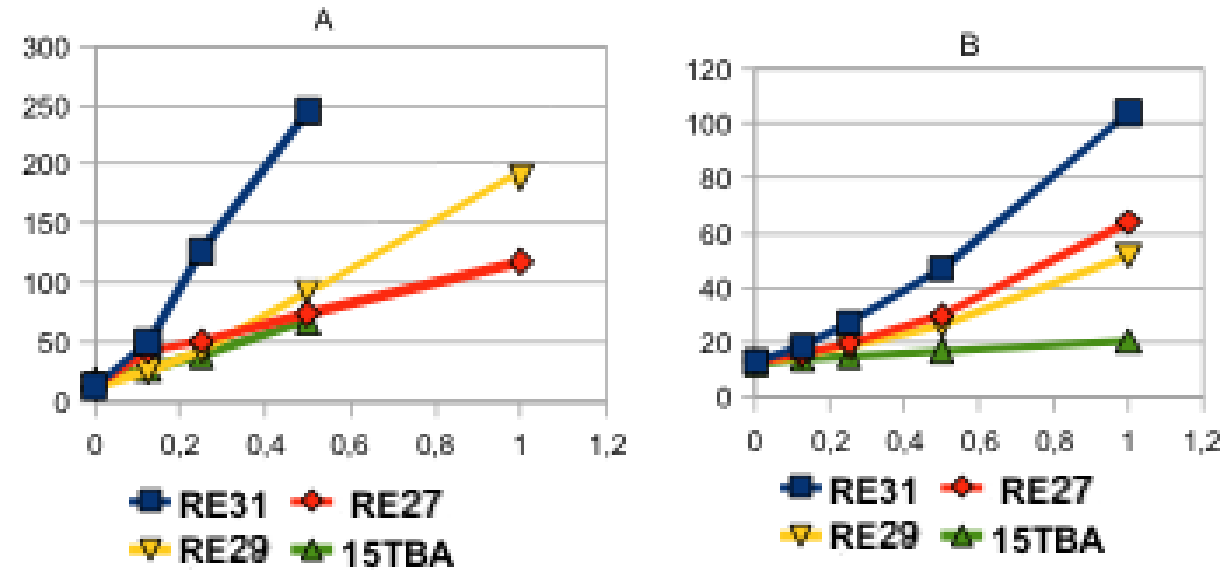

C

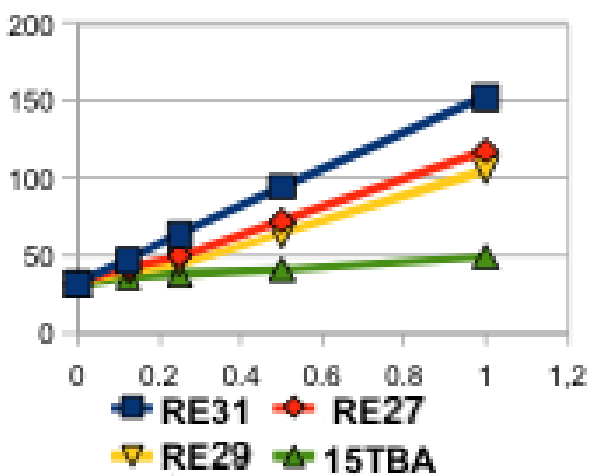

Aptamers: RE31 (blue), 31TBA (yellow), RE25 (red), 15TBA (green) were added in the specified concentrations to human plasma, and thrombin time (A), prothrombin time (B), and aPTT (C) were recorded in the presence of human thrombin. The time of fibrin clot formation has been recorded in all tests. The results of $3-4$ reproducible experiments are presented. On the $\mathrm{X}$ axis is the aptamer concentration $(\mu \mathrm{M})$, on the $\mathrm{Y}$ axis is the time (seconds).

Figure 2: Effects of RE31, 31TBA, and other aptamers on human plasma clotting formation. 

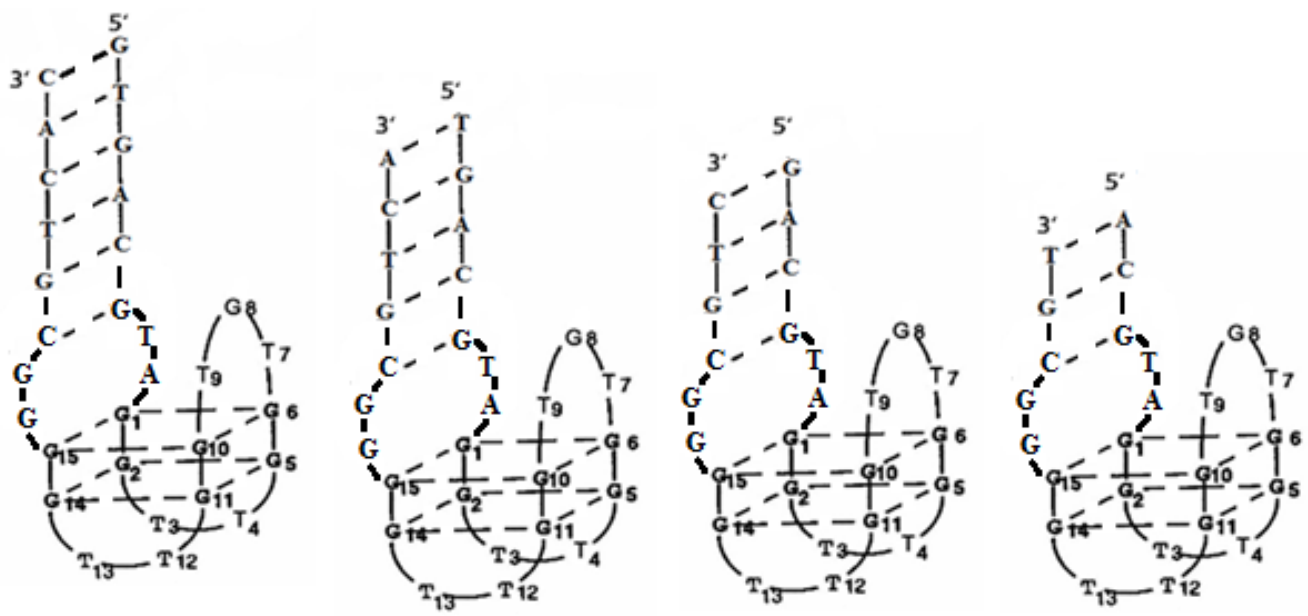

RE31 RE29 RE27 RE25
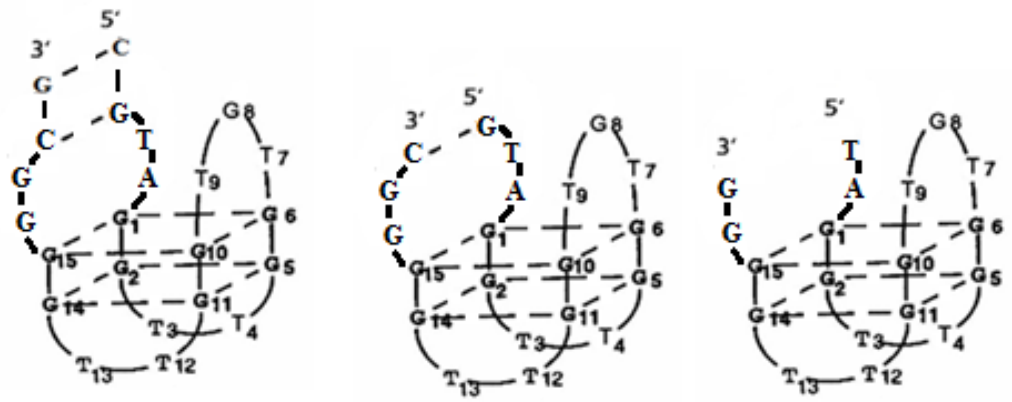

RE23 RE21 RE19

All the structures contain G-quadruplex domain. The aptamer molecules differ with duplex region.

Figure 3: The proposed tertiary structures of the aptamers RE31, RE29, RE27, RE25, RE23, RE21, RE19 (left to right).

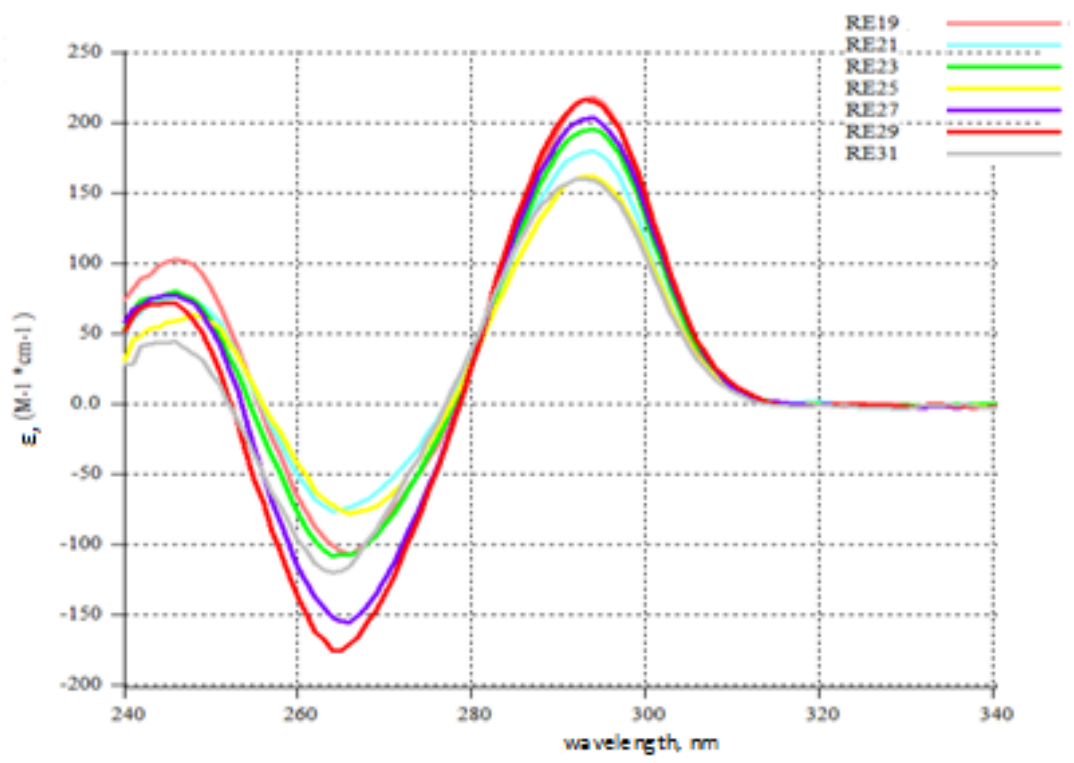

Figure 4: CD spectra of aptamers 15TBA, RE19, RE21, RE23, RE25, RE27, RE29, RE31 in a buffer, containing $20 \mathrm{mM}$ Tris- $\mathrm{HCl}, \mathrm{pH}=7,2 ; 5 \mathrm{mM} \mathrm{KCl}$ at $4{ }^{\circ} \mathrm{C}$. $15 \mathrm{TBA}$

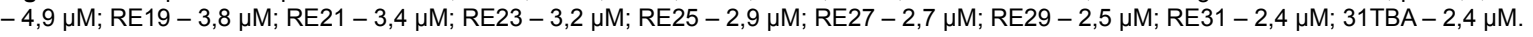




\begin{tabular}{|l|l|l|l|l|l|l|l|} 
15TBA & RE19 & RE21 & RE23 & RE25 & RE27 & RE29 & RE31 \\
\hline
\end{tabular}

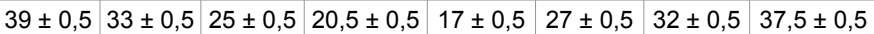

The CD values were recorded at $294 \mathrm{~nm}$, melting temperatures are in ${ }^{\circ} \mathrm{C}$. The attachment of additional oligonucleotide sequences to the G-quadruplex destabilizes its structure. On the other hand, the greater the number of complementary pairs formed by these sequences, the higher the stability of the G-quadruplex domain in a given aptamer. This domain is the least stable in aptamer RE25, where five pairs of nucleotides are attached to it. Three of them can form a duplex, but its stability is low.

Table 1: Melting temperatures of the aptamers, detecting by Circular dichroism.

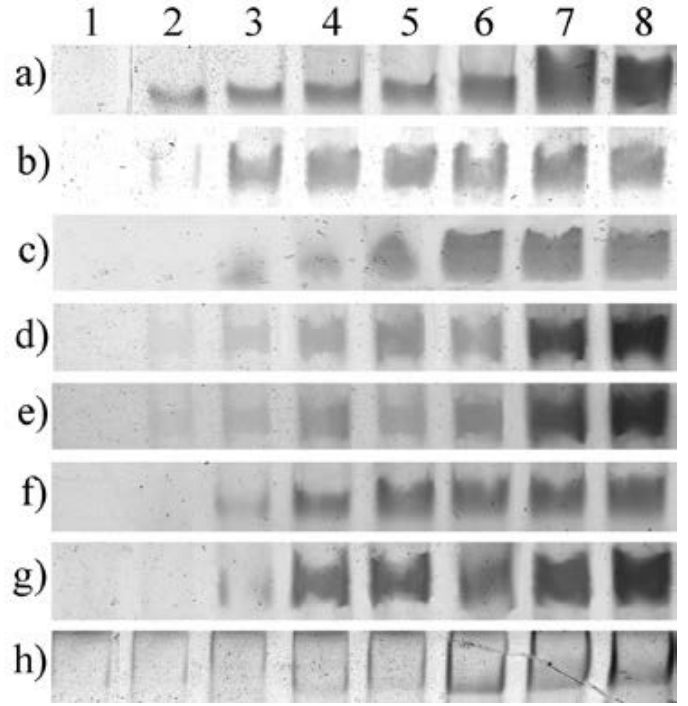

Figure 5: The thrombin-aptamer complexes, fixed in non-denaturating $8 \%$ PAGE.

The gel fragments are presented for ease of comparison.

a) RE31 - $100 \mathrm{nM} ; 1-0 ; 2-40 \mathrm{nM} ; 3-60 \mathrm{nM} ; 4-80 \mathrm{nM} ; 5-100 \mathrm{nM}$ $6-120 \mathrm{nM} ; 7-140 \mathrm{nM} ; 8-160 \mathrm{nM}$ thrombin;

b) RE29-200 nM; $1-0 ; 2-40 \mathrm{nM} ; 3-60 \mathrm{nM} ; 4-80 \mathrm{nM} ; 5-100 \mathrm{nM}$; $6-120 \mathrm{nM} ; 7-140 \mathrm{nM} ; 8-160 \mathrm{nM}$ thrombin;

c) RE27-200 nM; $1-0 ; 2-40 \mathrm{nM} ; 3-60 \mathrm{nM} ; 4-80 \mathrm{nM} ; 5-100 \mathrm{nM}$ $6-120 \mathrm{nM} ; 7-140 \mathrm{nM} ; 8-160 \mathrm{nM}$ thrombin;

d) RE25- $200 \mathrm{nM} ; 1-0 ; 2-120 \mathrm{nM} ; 3-180 \mathrm{nM} ; 4-240 \mathrm{nM} ; 5-300$ $\mathrm{nM} ; 6-360 \mathrm{nM} ; 7-400 \mathrm{nM} ; 8-400 \mathrm{nM}$ thrombin;

e) R23 - $200 \mathrm{nM} ; 1-0 ; 2-120 \mathrm{nM} ; 3-180 \mathrm{nM} ; 4-240 \mathrm{nM} ; 5-300$ $\mathrm{nM} ; 6-360 \mathrm{nM} ; 7-400 \mathrm{nM} ; 8-400 \mathrm{nM}$ thrombin;

f) RE21 - $200 \mathrm{nM} ; 1-0 ; 2-120 \mathrm{nM} ; 3-180 \mathrm{nM} ; 4-240 \mathrm{nM} ; 5-300$ $\mathrm{nM} ; 6-360 \mathrm{nM} ; 7-400 \mathrm{nM} ; 8-400 \mathrm{nM}$ thrombin;

g) $\mathrm{RE} 19-250 \mathrm{nM} ; 1-0 ; 2-120 \mathrm{nM} ; 3-180 \mathrm{nM} ; 4-240 \mathrm{nM} ; 5-300$ $\mathrm{nM} ; 6$ - $360 \mathrm{nM} ; 7$ - $400 \mathrm{nM} ; 8-400 \mathrm{nM}$ thrombin;

h) $\mathrm{RE} 15-300 \mathrm{nM} ; 1-0 ; 2-240 \mathrm{nM} ; 3-360 \mathrm{nM} ; 4-480 \mathrm{nM} ; 5-600$ $\mathrm{nM} ; 6-720 \mathrm{nM} ; 7-840 \mathrm{nM} ; 8-960 \mathrm{nM}$ thrombin;

\begin{tabular}{|c|c|c|c|c|}
\hline aptamer & 15TBA & RE19 & RE21 & RE23 \\
\hline & $55,2 \pm 3,4$ & $79,1 \pm 2,6$ & $168,9 \pm 16,0$ & $200,8 \pm 16,5$ \\
\hline aptamer & RE25 & RE27 & RE29 & RE31 \\
\hline & $294,1 \pm 32,8$ & $41,5 \pm 2,1$ & $28,3 \pm 3,5$ & $7,2 \pm 2,5$ \\
\hline
\end{tabular}

Initial data were plotted as binding-curves, and linearized in Scatchard's coordinates.

The $\mathrm{K}_{\mathrm{d}}$ increases in a row from RE19 to RE25 and then dramatically decreases (from RE27 to RE31).

The $\mathrm{K}_{d}$ value characterizes the stability of the thrombin-aptamer complex.

Table 2: Apparent dissociation constants $K_{d}(n M)$ of the thrombin-aptamer complexes (based on PAGE data).

Scatchard's coordinates, and the appeared dissociation constants were calculated from the tangent and half-height of each plot.

Table 2 shows the appeared dissociation constants of all complexes obtained in this study. It is obvious that the affinity of aptamers to thrombin correlates with their thermostability. The dissociation constants increase (i.e., the affinity decreases) in the series from 15TBA to RE25 and then decrease again; i.e., the affinity to thrombin increases in the series RE27 < RE29 < RE31. Thus, aptamer RE31 is the most effective in binding thrombin.

Moreover, the affinity of aptamers to thrombin shows a direct correlation with the stability of their quadruplex domain: the more thermostable the quadruplex, the higher the affinity. Thus, aptamer RE25 is characterized by the lowest melting temperature of the G-quadruplex, and its complexes with thrombin have a very high dissociation constant $(294.1 \pm 32.8 \mathrm{nM})$, which is evidence for their extreme instability. On the other hand, aptamers RE27, RE29, and RE31 form increasingly stable complexes, with the respective dissociation constants being $41.5 \pm 2.1,28.3 \pm 3.5$, and $7.2 \pm 2.5 \mathrm{nM}$.

It appears that the presence of additional nucleotides (the duplex domain) markedly improves the affinity of aptamers to thrombin, with a 6-bp duplex sequence contributing to aptamer-thrombin binding, probably by providing additional contacts between these molecules.

The CD spectra of all aptamers have a characteristic peak at 294 $\mathrm{nm}$ and share a common isosbestic point, which indicates that the mechanism of G-quadruplex binding to thrombin is the same in all cases. The height of this peak decreases in aptamers containing longer duplex domains, which may be evidence for loosening of the G-quadruplex structure; on the other hand, the presence of such a domain provides for better contact between the thrombin and aptamer molecules, which is reflected in TT and PT values. It may be concluded that the G-quadruplex is the functional unit in the aptamer-thrombin interaction, while the duplex domain of the aptamer aids in the "adjustment" of its conformation to that of the protein molecule. Our conclusion is supported by recent data of Russo Kraus et al. [20] that duplex-quadruplex motifs play a special structural role in thrombin binding by DNA aptamer [20]. In particular, these authors have shown that the overall shape of the molecule with such motifs allows both of them to interact with thrombin.

\section{Acknowledgements}

This work was supported by the Russian Foundation for Basic Research, grants No 11-04-01530.

\section{References}

1. Bock LC, Griffin LC, Latham JA, Vermaas EH, Toole JJ (1992) Selection of single-stranded DNA molecules that bind and inhibit human thrombin. Nature 355: 564-566.

2. Tuerk C, Gold L (1990) Systematic evolution of ligands by exponential enrichment: RNA ligands to bacteriophage T4 DNA polymerase. Science 249 : 505-510.

3. Ellington AD, Szostak JW (1990) In vitro selection of RNA molecules that bind specific ligands. Nature 346: 818-822.

4. Tasset DM, Kubik MF, Steiner W (1997) Oligonucleotide inhibitors of human thrombin that bind distinct epitopes. J Mol Biol 272: 688-698.

5. Macaya RF, Schultze P, Smith FW, Roe JA, Feigon J (1993) Thrombin-binding DNA aptamer forms a unimolecular quadruplex structure in solution. Proc Natl Acad Sci U S A 90: 3745-3749.

6. Wang KY, McCurdy S, Shea RG, Swaminathan S, Bolton PH (1993) A DNA aptamer which binds to and inhibits thrombin exhibits a new structural motif for DNA. Biochemistry 32: 1899-1904.

7. Nimjee SM, Rusconi CP, Harrington RA, Sullenger BA (2005) The potential of aptamers as anticoagulants. Trends Cardiovasc Med 15: 41-45.

8. Brody EN, Gold L (2000) Aptamers as therapeutic and diagnostic agents. J Biotechnol 74: 5-13. 
Citation: Spiridonova VA, Glinkina KA, Gainutdinov AA, Arutyunyan AM (2014) Production of Thrombin Complexes with DNA Aptamers Containing G-Quadruplex and Different Duplexes. J Nephrol Ther 4: 149. doi:10.4172/2161-0959.1000149

9. Proske D, Blank M, Buhmann R, Resch A (2005) Aptamers--basic research, drug development, and clinical applications. Appl Microbiol Biotechnol 69: $367-$ 374

10. Spiridonova VA (2010) [Molecular recognition elements--DNA/RNA-aptamers to proteins]. Biomed Khim 56: 639-656.

11. Padmanabhan K, Padmanabhan KP, Ferrara JD, Sadler JE, Tulinsky A (1993) The structure of alpha-thrombin inhibited by a 15-mer single-stranded DNA aptamer. J Biol Chem 268: 17651-17654.

12. Russo Krauss I, Merlino A, Giancola C, Randazzo A, Mazzarella L, et al. (2011) Thrombin-aptamer recognition: a revealed ambiguity. Nucleic Acids Res 39 7858-7867.

13. Russo Krauss I, Merlino A, Randazzo A, Novellino E, Mazzarella L, et al. (2012) High-resolution structures of two complexes between thrombin and thrombinbinding aptamer shed light on the role of cations in the aptamer inhibitory activity. Nucleic Acids Res 40: 8119-8128.

14. Macaya RF, Waldron JA, Beutel BA, Gao H, Joesten ME, et al. (1995) Structura and functional characterization of potent antithrombotic oligonucleotides possessing both quadruplex and duplex motifs. Biochemistry 34: 4478-4492.

15. Ikebukuro K, Okumura Y, Sumikura K, Karube I (2005) A novel method of screening thrombin-inhibiting DNA aptamers using an evolution-mimicking algorithm. Nucleic Acids Res 33: e108.

16. Dolinnaya NG, Yuminova AV, Spiridonova VA, Arutyunyan AM, Kopylov AM (2012) Coexistence of G-quadruplex and duplex domains within the secondary structure of 31-mer DNA thrombin-binding aptamer. J Biomol Struct Dyn 30: 524-531.

17. Dobrovolsky AB, Titaeva EV, Khaspekova SG, Spiridonova VA, Kopylov AM et al. (2009) Inhibition of thrombin activity with DNA-aptamers. Bull Exp Bio Med 148: 33-36.

18. Mazurov AV, Titaeva EV, Khaspekova SG, Storojilova AN, Spiridonova VA, et al. (2011) Characteristics of a new DNA aptamer, direct inhibitor of thrombin. Bull Exp Biol Med 150: 422-425.

19. Maniatis T, Fritsch E, Sambrook J (1982) Molecular cloning: a labolatory manual. Cold spring harbor laboratory press.

20. Russo Krauss I, Pica A, Merlino A, Mazarella L, Sica F (2013) Duplexquadruplex motifs in a peculiar structural organization cooperatively contribute to thrombin binding of a DNA aptamer, Acta Crystallograph. Section D, D69: 2403-2411. 